\title{
Animation Key-Frame Extraction and Simplification Using Deformation Analysis
}

\author{
Tong-Yee Lee, Chao-Hung Lin, Yu-Shuen Wang, and Tai-Guang Chen
}

\begin{abstract}
Three-dimensional animating meshes have been widely used in the computer graphics and video game industries. Reducing the animating mesh complexity is a common way of overcoming the rendering limitation or network bandwidth. Thus, we present a compact representation for animating meshes based on novel key-frames extraction and animating mesh simplification approaches. In contrast to the general simplification and key-frames extraction approaches which are driven by geometry metrics, the proposed methods are based on a deformation analysis of animating mesh to preserve both the geometric features and motion characteristics. These two approaches can produce a very compact animation representation in spatial and temporal domains, and therefore they can be beneficial in many applications such as progressive animation transmission and animation segmentation and transferring.
\end{abstract}

Index Terms-Animating meshes, deformation analysis, key-frame extraction, simplification.

\section{INTRODUCTION}

$\mathbf{R}$ ECENTLY, many researchers have preferred representing animation using a sequence of deforming meshes (i.e., time-varying meshes) to satisfy the high-quality skin detail requirement [1], [2]. High-resolution deforming meshes with a great number of frames are required to represent pleasing and realistic animations. In this paper, we present novel methods for animation simplification and key-frame extraction. With the proposed approach, many redundant frames (i.e., those that can be interpolated by other frames) and perceptually meaningless animation data can be omitted or reduced, therefore greatly reducing the animation data requirement. Many important applications can also benefit from this novel approach, which will be demonstrated in this paper.

The aim of key-frame extraction is to search for good keyframes for reconstructing all other frames in an animation. The number of key-frames should be as small as possible, and the quality of the reconstructed frames should be as high as possible.

Manuscript received December 14, 2006; revised June 1, 2007 and July 14, 2007. This paper was supported by the National Science Council, Taiwan, R.O.C., under Contract NSC-95-2221-E-006-368, Contract NSC-95-2221-E-006-193-MY2, Contract NSC-96-2628-E-006-200-MY3, Contract NSC-96-2221-E-006-312 -MY2, and in part by the Landmark Program of the NCKU Top University Project under Contract B0008 and C0038. This paper was recommended by Associate Editor L. Onural.

T.-Y. Lee, Y.-S. Wang, and T.-G. Chen are with the Computer Science and Information Engineering Department, National Cheng-Kung University, Tainan 701, Taiwan, R.O.C. (e-mail: tonylee@mail.ncku.edu.tw; braveheart@csie.ncku.edu.tw; taiguang@csie.ncku.edu.tw).

C.-H. Lin is with the Geomatics Department, National Cheng-Kung University, Tainan 701, Taiwan, R.O.C. (e-mail: linhung@mail.ncku.edu.tw).

Color versions of one or more of the figures in this paper are available online at http://ieeexplore.iee.org.

Digital Object Identifier 10.1109/TCSVT.2008.918456
Generally, the animation key-frame extraction can be solved as a key-frame search optimization problem. However, the search space is large (i.e., large number of frames) and this search space is not perfectly smooth (i.e., the number of key-frames is not determined a priori). To solve these problems, we propose a deformation-driven genetic algorithm (GA) to efficiently search optimal key-frames from a sequence of animating meshes.

In the literature, many mesh simplification approaches have been proposed to simplify static meshes such as [3], [4]. However, naïvely simplifying animating meshes using these methods cannot guarantee preserving both the geometric and the motion features of the animating meshes. These static techniques can guarantee simplifying a single frame with good geometric feature fidelity. Simplifying any key-frame in animating meshes may eliminate the important details required to represent other frames. To better simplify animating meshes, the proposed method will analyze the deforming information presented in the entire animating sequence.

The major contributions of the proposed methods for animation key-frame extraction and simplification are listed here.

- A deformation-driven GA is proposed to efficiently search good representative animation key-frames. These extracted key-frames can reconstruct other frames with good quality.

- A novel mesh simplification method based on animating mesh deformation information is proposed. With this new approach, the characteristics of animating meshes in both spatial and temporal domains can be successfully preserved. This method generates a sequence of simplified animating meshes with a static connectivity. Therefore, the annoying popping effect caused by changing connectivity/geometry is automatically avoided.

Many important applications such as animation segmentation and transferring can benefit from the proposed methods. Experimental evaluations of these applications are demonstrated and compared with other previous schemes.

\section{RELATED WORK}

There have been many methods proposed for animation keyframe extraction and simplification. We describe only the most related schemes for animating mesh in this section.

\section{A. Key-Frame Extraction}

Similar to video summarization [5]-[7], animation key-frame or key-posture extraction is the process of selecting meaningful frames from time-varying data. The related works can be categorized into curve simplification, clustering, and matrix factorization [8] based on to which domain the key-frame extraction problem will be transferred. In [9], the animation data is 
considered as a trajectory curve in a high dimensional space. The key-frame extraction is converted into a curve simplification problem. In [10], the key-frame extraction becomes a clustering problem that attempts to group frames with similar posture. Once the key-frames are extracted, the remaining animation frames can be reconstructed by a linear combination of the extracted key-frames. In the category of matrix factorization [8], an animation is represented as a matrix formed by placing all of the vertices of a frame in a row. This matrix is then approximately factorized into a weight matrix and a key-frame matrix. For example, in [8]-[10], the optimal solution to the key-frame search is obtained under similar constraints or objective functions. However, these approaches are very time-consuming and not efficient for practical applications. To the best of our knowledge, little research work has addressed this issue. There are two factors that make this problem more challenging. First, the high-resolution animation sequence usually consists of a large number of frames (above 100 frames). Second, the optimal number of extracted key-frames cannot be determined a priori. Therefore, an efficient search approach is required. We introduce the deformation-driven GA to efficiently search good representative animation key-frames. Once the key-frames are extracted, similar to [8] and [10], the animation is reconstructed by a linear combination of the extracted key-frames for better approximation.

\section{B. Animation Mesh Simplification}

The previous methods can be grouped into two categories: static connectivity and dynamic connectivity [11], [12]. Many researchers [11]-[14] proposed adaptively changing the connectivity and dynamically improving the quality of the resulting simplified meshes. To create an appealing simplified mesh at each frame, a great amount of connectivity updating is required, thereby usually causing the unpleasant popping effect. These previous methods attempted to reduce this problem by maintaining both temporal and spatial coherence in updating the connectivity. However, the popping still could not be eliminated. In practice, a small number of popping effects will make an animation unpleasant. In the static connectivity category, several methods [15], [16] extend the standard static mesh simplification (i.e., QSlim algorithm [3]) to take all of the frames of an animation into account when choosing a collapsing-edge candidate. All simplified animating meshes are represented by a single connectivity, and therefore the popping effect caused by changing connectivity/geometry is automatically avoided. The saliency algorithm presented in [25] is used in mesh simplification [26]. In [26], the authors modify the QSlim algorithm by weighing the quadric error with a salient value. The purpose is to preserve the salient regions in the simplification process. This approach can be easily extended to simplify time-varying meshes using the approach presented in [15]. However, this approach may lose some geometric details due to the over-preservation of geometric features. The major challenges of simplifying time-varying meshes are maintaining both the geometric features and the motion characteristics of an animation. To solve these two difficulties, we incorporate the temporal information for the simplification of time-varying meshes. We select the collapsing-edge candidates using the metric of weighing the quadric geometrical error with the deformation degree. In this manner, the geometric features can be preserved by the quadric error metric, and the motion characteristics can be preserved by the deformation degree metric.

\section{DEFormation ANALYSIS}

Deformation analysis measures a triangle deformation degree which describes how significant motion behaves in this triangle in an animation. For this analysis, we measure the difference of the deformation gradient [1], called the deformation distance, between any two adjacent triangles. Recently, the deformation gradient has been applied to various computer graphic applications including deformation transferring [1], animating mesh segmentation [17], [18], and mesh deformation [19]. The deformation gradient is a nontranslation affine transformation between two triangle orientation matrices. Basically, a deforming mesh with $n$ frames is represented as $A=\left\{M^{t}\right\}, 1 \leq t \leq n$, where $M^{t}$ represents the deforming mesh in the frame $t$ and each $M^{t}$ consists of many triangles called $f_{i}^{t}$ s. Each face $f_{i}^{t}$ consists of three vertices $v_{1}^{t}, v_{2}^{t}$, and $v_{3}^{t}$. The orientation matrix $O_{i}^{t}$ of $f_{i}^{t}$ can be computed by $\left[v_{2}^{t}-v_{1}^{t} v_{3}^{t}-v_{1}^{t} v_{4}^{t}-v_{1}^{t}\right]$, where $v_{4}^{t}=v_{1}^{t}+\left(v_{2}^{t}-v_{1}^{t}\right) \times\left(v_{3}^{t}-v_{1}^{t}\right) / \sqrt{\left|\left(v_{2}^{t}-v_{1}^{t}\right) \times\left(v_{3}^{t}-v_{1}^{t}\right)\right|}$. Similarly, the orientation matrix of the same face in the reference frame $r$ denoted as $f_{i}^{r}$ is $O_{i}^{r}=\left[v_{2}^{r}-v_{1}^{r} v_{3}^{r}-v_{1}^{r} v_{4}^{r}-v_{1}^{r}\right]$. Usually, the first frame is selected as the reference frame. The nontranslation affine transformation, called deformation gradient $D_{i}^{t}$, between $f_{i}^{t}$ and $f_{i}^{r}$ can then be computed using

$$
D_{i}^{t}=O_{i}^{t}\left(O_{i}^{r}\right)^{-1}
$$

The differential deformation gradient between two adjacent faces $f_{i}^{t}$ and $f_{j}^{t}$ at time frame $t$ can be measured using the matrix subtraction $D_{i}^{t}-D_{j}^{t}$. We set the Frobenius norm of this differential deformation gradient as the deformation distance between these two faces, that is

$$
D D_{i, j}^{t}=\left\|D_{i}^{t}-D_{j}^{t}\right\|_{F} .
$$

We compute the deformation distances between the adjacent faces at each frame. The largest one denoted as $D D_{i, j}$ is chosen to represent the deformation degree between two adjacent faces among all of the frames in an animation. To define the deformation degree of a face $p$, we simply average the deformation degrees between one face $f_{i}$ and its neighboring faces $f_{k}$, that is

$$
\text { DDegree }_{f_{i}}=\underset{f_{k} \in \operatorname{Adjacency}\left(f_{i}\right)}{\operatorname{avg}}\left(D D_{i, k}\right) .
$$

The deformation degree of $f_{i}$ will be comparatively large if the region around this face has a very different motion. Otherwise, the deformation degree will be small if this region has a similar motion. Therefore, the larger face deformation degree of $f_{i}$ implies a more important motion feature in the animation behaving on this face. We use the face deformation degree as a metric to simplify animating meshes and extract animation key-frames. 


\section{Simplifying DEFORMING Mesh}

The goal of our simplification approach is to simplify the deforming mesh under the minimizing animation distortion constraint. The proposed method is based on the QSlim algorithm [3] which introduces the quadric error metric (QEM) for collapsed-edge selection. The QSlim algorithm formulates the vertex distortion cost as the quadric distances from the vertex to its adjacent planes. This approach is originally designed to simplify a static mesh. To simplify a deforming mesh, we extend this distortion cost as the quadric distances from the vertex to its adjacent faces weighted by the face deformation degree described in (3). Therefore, the distortion cost of vertex $v^{f}$ at the time frame $f$ is represented as follows:

$$
d\left(v^{f}\right)=v^{f^{T}}\left(\sum_{p \in \operatorname{planes}\left(v^{f}\right)}\left(p p^{T} * \text { DDegree }_{p}\right)\right) v^{f}
$$

where $p$ represents the plane (face) adjacent to the vertex.

Like [3], the vertex quadric $Q_{v}^{f}$ is defined as the sum of the outer product of the face adjacent to vertex $v^{f}$ multiplied by this face deformation degree

$$
Q_{v}^{f}=\sum_{p \in \operatorname{planes}\left(v^{f}\right)}\left(p p^{T} * \text { DDegree }_{p}\right) .
$$

Initially, the distortion cost $d(v)$ will be zero because the adjacent faces pass through this vertex. When the two vertices $v_{1}^{f}$ and $v_{2}^{f}$ are contracted to a new vertex $v_{k}^{f}$, the quadric error of this new vertex conforms to the additive rule $Q_{v_{k}}^{f}=Q_{v 1}^{f}+Q_{v 2}^{f}$. Therefore, the edge-collapse cost of an edge $\left(v_{1}^{f}, v_{2}^{f}\right)$ during the iterative contraction is formulated as follows:

$$
\cos t^{f}\left(v_{1}^{f}, v_{2}^{f}\right)=v_{k}^{f^{T}}\left(Q_{v 1}^{f}+Q_{v 2}^{f}\right) v_{k}^{f} .
$$

Note that the new vertex $v_{k}$ can be directly solved by optimizing the vertex position frame by frame under the constraint $\min _{v_{k}} \cos t^{f}\left(v_{1}^{f}, v_{2}^{f}\right)$ as discussed in [3].

For a deforming mesh, the edge-collapse cost of an edge $\left(v_{1}, v_{2}\right)$ can be simply formulated as the sum of edge-collapse cost at all time frames, that is,

$$
\operatorname{Cost}\left(v_{1}, v_{2}\right)=\sum_{f \in \text { Frames }} \operatorname{Cost}^{f}\left(v_{1}^{f}, v_{2}^{f}\right) .
$$

As mentioned in Section II, to avoid the popping effects caused by the connectivity dynamic update, our simplification method prefers a single connectivity to represent all animating meshes in the animation. As mentioned in [11], [12], when the mesh deformation is highly nonrigid, the static connectivity usually leads to bad simplification to certain frames. The measurement of the proposed edge-collapse cost considers both the geometric and the motion features. Therefore, the proposed method will not suffer from this problem. The experimental results will show that our method performs well for both rigid and nonrigid mesh animation.

\section{ANIMATION KEY-FrAmE EXTRACTION}

Here, we propose a deformation-driven GA method to fastsearch animation key-frames.

\section{1}

$1:$ Keyframe

$0:$ Non-keyframe

Fig. 1. Example of a key-frame sequence encoding.

\begin{tabular}{|c|c|}
\hline 101010101010101 \\
\hline $120 \%$ \\
\hline 101010101010101 \\
\hline 110100001001001 \\
\hline$\vdots$ \\
$\vdots 80 \%$ \\
\hline 1010101010101 \\
\hline
\end{tabular}

(a)

(b)

Fig. 2. (a) Chromosome with "1" bit regular interleaving. (b) Initial population.

\section{A. Extracted Key-Frames Encoding}

For any search and learning method, the candidate solution encoding method is very vital to the success of a genetic algorithm. Binary encoding is the most common and simplest method. We use fixed-length and fixed-order binary coding, namely, a bit string, to represent a candidate key-frame sequence as a chromosome. Each frame is encoded as a single bit. The " 1 "-bit represents a key-frame and the " 0 "-bit represents a non-key-frame. In this manner, each key-frame sequence is represented as a bit string. The length of a bit string is equal to the number of animation frames and the encoding order is from left-to-right (i.e., from the first frame to the last frame). Fig. 1 shows an example of a key-frame sequence encoding.

\section{B. Population Initialization}

Theoretically, the initial population (i.e., bit strings) is chosen randomly in order to maintain a wide chromosome spectrum. However, from our experimental practice, this simple manner is not good enough in terms of the efficiency since an animation is usually a smooth sequence. The adjacent frames are similar. Therefore, the adjacent frames are usually not selected to be the key-frames at the same time. A more appropriate setting for an initial chromosome is a bit string with " 1 " bit regular interleaving, as shown in Fig. 2(a). To keep a wide spectrum of the chromosomes while considering the algorithm efficiency, we set $80 \%$ of the chromosomes in the initial population to be chosen randomly and the remaining $20 \%$ of chromosomes set as bit strings with the " 1 " bit regularly interleaving. Fig. 2(b) shows an example of our initial population. The population size is set at 1000 in our experiments.

\section{Fitness Function}

The fitness function, or objective function, is used to evaluate the possibility of a chromosome for being the best key-frame extraction result. Each chromosome in the current population will be assigned a score (fitness) which represents how well that chromosome represents the key-frames of a given animation. From the key-frame extraction point of view, the number of extracted key-frames must be as small as possible while the reconstructed animation error using these extracted key-frames must 
be minimized. Therefore, two factors are taken into account for key-frame extraction optimization: 1) the errors in the reconstructed animation and 2) the number of extracted key-frames. Therefore, the fitness function is defined by

$$
\operatorname{Fitness}(T)=\frac{1}{\alpha \times \operatorname{Error}(T)+(1-\alpha) \times \operatorname{Ratio}(T)}
$$

where the instance $T$ represents a candidate solution and $\alpha$ is a user-defined weight coefficient. $\operatorname{Ratio}(T)$ is the ratio of the number of selected key-frames over the number of animation frames.

In (8), the error term $\operatorname{Error}(T)$ is defined as the difference between the original animation and its corresponding reconstructed animation using extracted key-frames. To efficiently calculate this term, we simply adopt a liner interpolation to interpolate the non-key-frames from the extracted key-frames. We then use the following equation to calculate the error between two corresponding frames:

$$
\begin{aligned}
& \text { FrameError }\left(\text { frame }^{A}, \text { frame }^{B}\right) \\
& =\sum_{p \in \text { Faces }}\left(\beta \times \| \text { Deformation } p_{p^{A}, p^{B}} \|_{F}\right. \\
& \left.\quad+(1-\beta) \times \text { Distance }_{p^{A}, p^{B}}\right)
\end{aligned}
$$

where $\beta$ is a user-defined weight coefficient and frame $^{A}$ and frame $^{B}$ represent a frame in the original animation and its corresponding frame in the reconstructed animation, respectively.

The error function FrameError() is factorized into two terms: Deformation and Distance. The Deformation term is to measure the Frobenius norm of the deformation gradient [i.e., calculated by (1)], between a face $p^{A}$ in $f r a m e^{A}$ and its corresponding face $p^{B}$ in frame $^{B}$. The Distance term calculates the displacement of these two corresponding faces. It simply averages the displacement of three vertices in the face. Because the fitness function is factored into two distinctive terms, the error term $\operatorname{Error}(T)$ must be normalized. We assume that the worst key-frame extraction case, that is, the case with the maximum error, is only the first and last frames selected as key-frames. The intermediated frames are interpolated using only these two frames. In this situation, the reconstructed animation will have the maximum error. Under this assumption, the normalization process is calculated using

$$
\text { Error }=\frac{\text { NonKeyframeError }}{\text { MaximumError }}
$$

where the MaximumError represents the worst case error and the NonKeyframeError represents the candidate solution error (i.e., extracted key-frames).

In (8), the fitness function formed by two terms is a tradeoff between the reconstruction error and the number of key-frames. The fewer the number of key-frames selected, the greater the generated reconstructed error. Therefore, the fitness function is designed as a weighted combination of these two terms. The significance of these two terms can be controlled by a user-defined parameter $\alpha$.

\section{Selection}

After the encoding and the initial population are decided, the next step is to choose a chromosome in the population that will

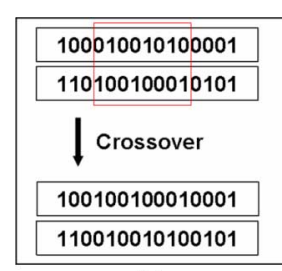

(a)

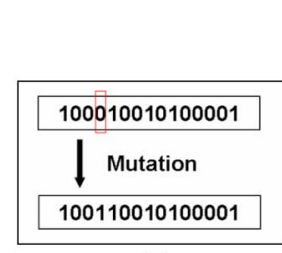

(b)

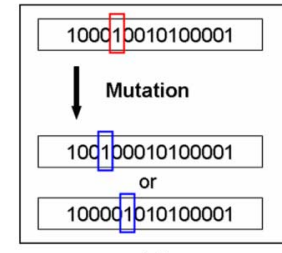

(c)
Fig. 3. Two GA operators and their offspring. (a) Crossover. (b) Mutation. (c) Offspring.

create offspring for the next generation. The purpose of selection is to emphasize the fitter chromosome in the population in hopes that their offspring will in turn have higher fitness. In this paper, we adopt the simplest and most popular one, that is, fitness-proportionate selection. The expected value of a chromosome (i.e., the expected number of times a chromosome will be selected to reproduce), is that chromosome's fitness calculated by the fitness function. In other words, a chromosome with a higher fitness value has a higher probability of being selected.

\section{E. Genetic Operators}

Once the parent chromosomes are selected, we use two evolution strategies to generate offspring for the next generation: Crossover and Mutation. These two operators are described in detail as follows.

- Crossover: A two-point crossover approach is adopted here. Two loci are randomly chosen and the segments between them in the parent chromosomes are then exchanged to form two offspring. Fig. 3(a) shows an example of this operation.

- Mutation: We propose two mutation approaches. The first approach randomly chooses the mutation position and then exchanges the bit value (i.e., " 1 " changes into " 0 " or " 0 " changes into "1"), as shown in Fig. 3(b). The second approach is that we first randomly select a "1" bit as a mutation locus and then shift this locus right or left one bit to generate the offspring, as shown in Fig. 3(c). The shift direction is also randomly decided. The purpose of this design is to perform locus position fine-tuning. A fitter keyframe may be located around a candidate key-frame. This mutation approach can speed up algorithm convergence.

How the mutation and crossover rates are set is very important. In our experiments, the crossover probability is set to $95 \%$ and the mutation probability is set to $5 \%$. In terms of mutation probability, the probability of the random selection is set to $97 \%$ (the first approach) and the probability of locus shifting is set to $3 \%$ (the second approach).

\section{F. Termination Condition}

A genetic algorithm is usually executed in an iterative manner. In general, in the last generation, there are many highly fit chromosomes in the population. Therefore, the termination condition is when $50 \%$ of the chromosomes in the population have the same fitness, the fittest one is the same during the last 100 iterations. 


\section{G. Animation Reconstruction}

Once key-frames are extracted from an animation, we can construct an approximate animation using these key-frames. In the paper, we adopt Huang et al.'s approach [8] to perform reconstruction. The basic idea is that each frame of an animation is regarded as a linear combination of extracted key-frames. Therefore, each frame is approximated by linearly blending the key-frames as defined as

$$
F_{\mathrm{orig}}^{i} \approx \sum_{j=1}^{m} w_{i j} F_{\mathrm{key}}^{j}, \text { for } i=1, \ldots, n
$$

where $w_{i j}$ is the weight of the $j$ th key-frame $\left(F_{\mathrm{key}}^{j}\right)$ for reconstructing $i$ th frame $\left(F_{\text {orig }}^{i}\right), n$ represents the number of frames, and $m$ represents the number of key-frames.

The quality of animation reconstructed by all key-frames is better than that reconstructed by local key-frames. The animation reconstruction space spanned by local key-frames is only a subspace of that spanned by all key-frames. Therefore, the frames interpolated by local key-frames also can be blended by all key-frames, but not vice versa. However, the global support may result in temporal aliasing. Fortunately, this problem can be greatly reduced by optimizing the blending weights in the purpose of minimizing the reconstruction distortion. To obtain optimum weights $w_{i j}$, we minimize the following equation:

$$
\arg \min _{w_{i j}}\left\|F_{\text {orig }}^{i}-\sum_{j=1}^{m} w_{i j} F_{\mathrm{key}}^{j}\right\|^{2}, \quad \text { for } i=1, \ldots, n
$$

Therefore, an animation sequence with $n$ frames can be represented by $m$ key-frames and its corresponding weights for all frames, that is

$$
\begin{array}{r}
\left\{\left(F_{\mathrm{key}}^{1}, w_{11}, \cdots, w_{n 1}\right),\left(F_{\mathrm{key}}^{2}, w_{12}, \cdots, w_{1 n}\right), \cdots,\right. \\
\left.\left(F_{\mathrm{key}}^{m}, w_{1 m}, \cdots, w_{n m}\right)\right\}
\end{array}
$$

In the key-frame extraction optimization process, we simply use linear interpolation to construct an animation. The reconstructed result using linear interpolation is good enough to evaluate the fitness value. Usually, the reconstruction quality using [8] is not significantly better (i.e., varying from $3 \%$ to $12 \%$ ) than that using simple linear interpolation. However, the computational cost is much higher than that in linear interpolation. The reconstruction is performed frequently in the optimization process. It is too time-consuming to frequently reconstruct a candidate solution using [8]. To take efficiency into account, we simply use linear interpolation in the optimization step. Then, once the key-frames are extracted, we use [8] to reconstruct the final animation for better quality. The number of key-frames is determined automatically in the proposed approach. We extract fewer key-frames in a near-rigid animation and extract more key-frames in an extremely nonrigid animation. If the input animation has extreme deformation between frames (it implies that each frame is a key-frame), we need another morphing/interpolation technique to interpolate more in-between frames for a more smooth animation. This problem is beyond the scope of this paper.
TABLE I

VARIOUS EXPERIMENTAL ANIMATION SEQUENCES

\begin{tabular}{ccccc}
\hline $\begin{array}{c}\text { Animation } \\
\text { sequence }\end{array}$ & $\begin{array}{c}\text { Rigid/Soft } \\
\text { Body }\end{array}$ & \#Vertex & \#Face & \#Frame \\
\hline Runner & rigid-body & 7502 & 15000 & 25 \\
Dancer & rigid-body & 7061 & 14118 & 201 \\
Chicken & rigid-body & 3030 & 5664 & 400 \\
Homer & rigid-body & 5103 & 10202 & 25 \\
Horse-Gallop & rigid-body & 8431 & 16843 & 48 \\
Horse-Collapse & soft-body & 8431 & 16843 & 53 \\
Cow & soft-body & 2904 & 5804 & 204 \\
\hline
\end{tabular}

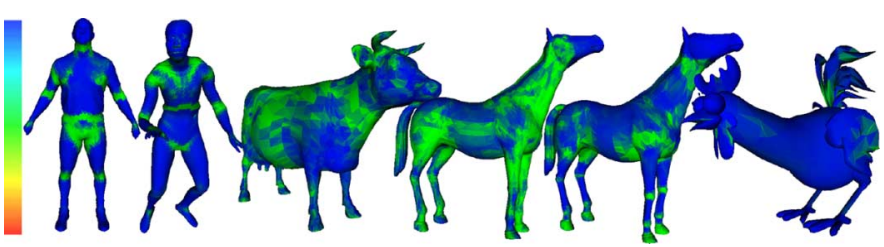

Fig. 4. Deformation analysis of various deforming mesh including Runner, Dancer, Cow, Horse-Collapse, Horse-Gallop, and Chicken. The deformation degree is coded by color ranging from red (the largest degree) to blue (the smallest degree).

\section{EXPERIMENTAL RESULTS AND DISCUSSIONS}

The various animation sequences used in the experiment are shown in Table I. To demonstrate the robustness and feasibility of the proposed animation key-frame extraction and simplification methods, both rigid-body and soft-body animation sequences were experimentally evaluated. The proposed methods are based on the deformation analysis. Therefore, we start with the visualization of the deformation analysis of various animation sequences in Fig. 4. Obviously, the faces lying around the joints have a larger deformation degree (green color). This means that these faces are significant motion features and must be preserved in the simplification process.

The experimental results were evaluated on an Intel Pentium 42.4 G PC with 1 G memory. The configuration of all parameters in the genetic algorithm was identical in all experiments (population size: 1000; crossover probability: 95\%; mutation probability: $5 \%$; terminate condition: $50 \%$ same chromosomes in the population and the fittest chromosome is unchanged during 100 iterations). These parameters were obtained from experiments (attempt to find the most suitable parameter setting). Some key-frame extraction results are shown in Figs. 5 and 6.

To evaluate the performance of the proposed key-frame extraction approach, we compare it using only a state-of-the-art method, KeyProbe [8], as follows. As for the other well-known approaches such as Curve Simplification [9], K-mean [20], ICA [21], and PCA [22], [23], a detailed discussion and comparison with [8] are provided in [8]. We adopted the commonly used peak signal-to-noise ratio (PSNR) to measure the reconstruction distortion. Similar to [8], we restrict the number of extracted key-frames to $10,20,30,40$, and 50 in the extraction process, that is, parameter $\alpha$ is set to 1.0 in (8). For these selected cases, we calculate the minimum, maximum, and average PSNR over 

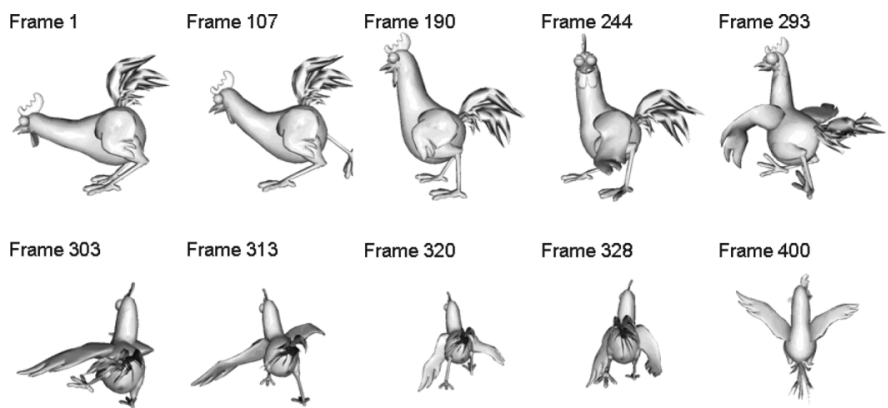

Frame 328
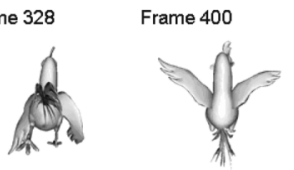

Fig. 5. Selected key-frames of Chicken animation.
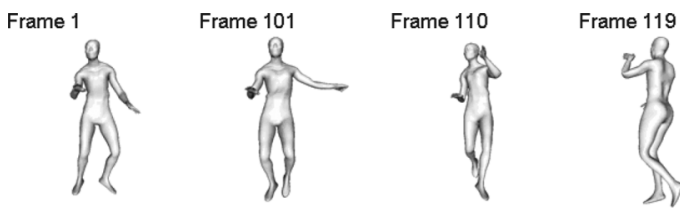

Frame 157

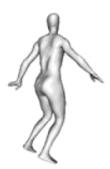

Frame 168

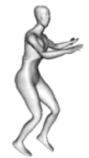

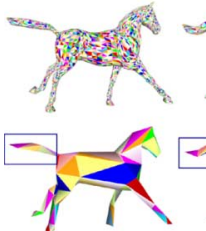
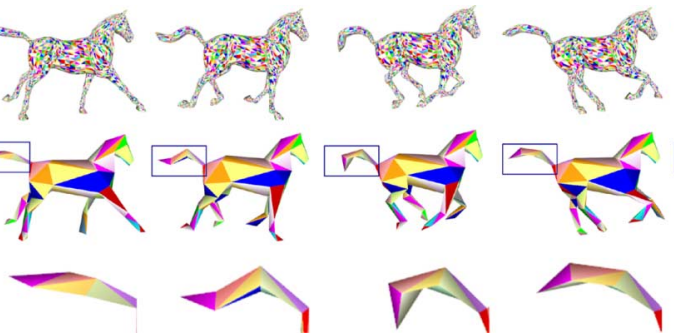

Fig. 7. Deforming mesh simplification. The deforming mesh is simplified from 8431 vertices (original) to $84(1 \%)$ vertices
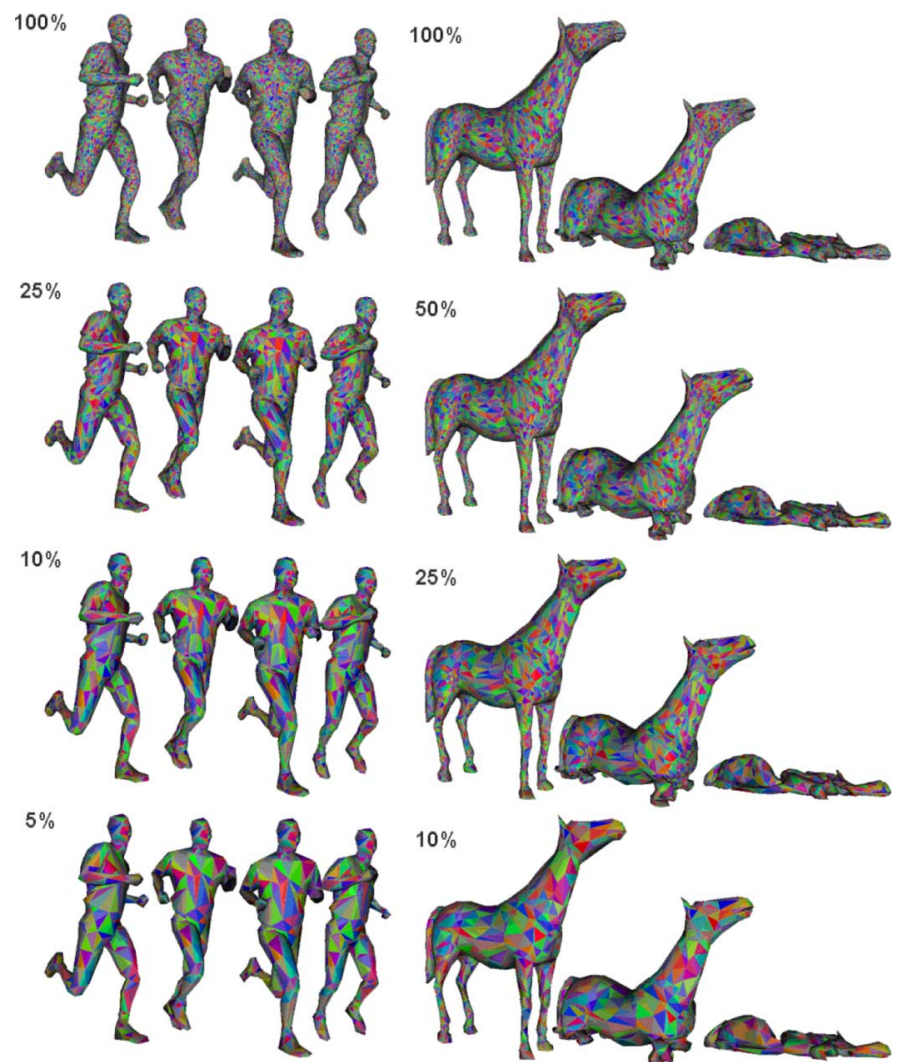

Fig. 8. Deforming mesh simplification.

approach than the time-consuming KeyProbe to extract an animation with a large number of frames.

For animation simplification, we first demonstrate the ability of motion feature preservation in Fig. 7. The proposed method can successfully preserve the tail motion details, as shown in Fig. 7 (the blue quadrilateral), while preserving the geometric appearance. This is because the faces around the joints of the tail have a larger deformation degree. They are recognized as significant motion features and are assigned a lower priority for the collapsed-edge selection. Therefore, the proposed method can preserve both the geometry and motion features in the simplification process. Fig. 8 shows the other experimental results. On the left-hand side of Fig. 8, the runner animation is simplified from 7502 vertices (original) to 1875 (25\%), to $750(10 \%)$, and finally to 375 (5\%) vertices. From this figure, the proposed approach can preserve an animation appearance of both geometry and motion, even in the most simplified case $(5 \%)$. On the right-hand side of Fig. 8, the collapsing takes about a half an hour. Therefore, our technique is a better 

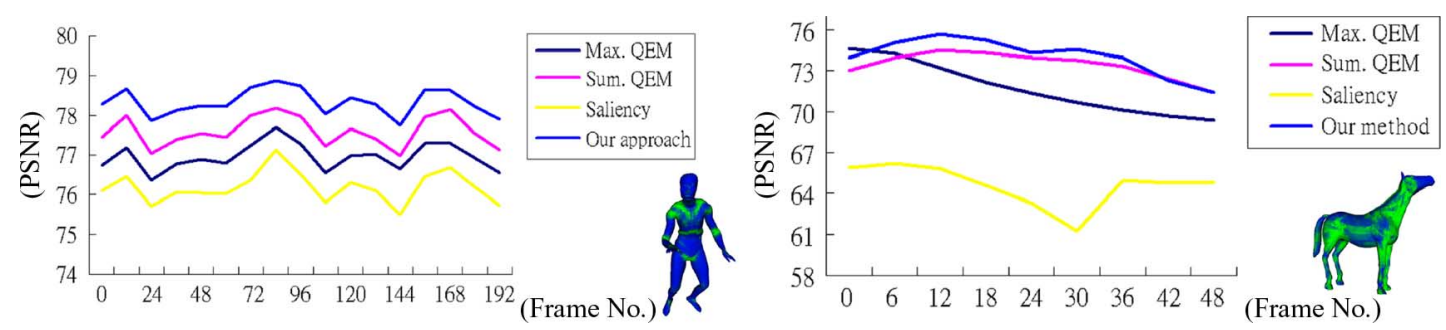

Fig. 9. Quality comparison between the Max. QEM, Sum. QEM [15], Saliency [26] and the proposed method.

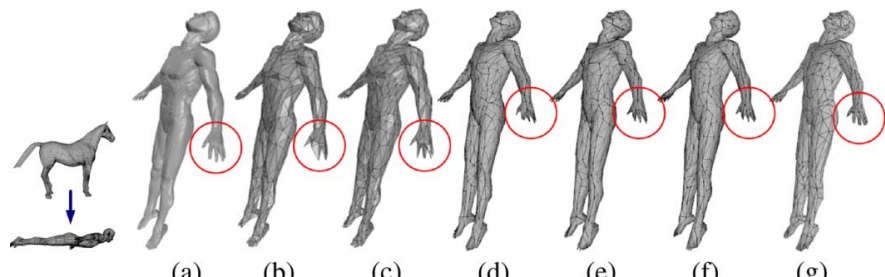

(a) (c) (e) (g) animation (first frame). (b) [11]. (c) [12]. (d) Max. QEM; (e) Sum. QEM [15]. (f) Saliency [26]. (g) Our method. Note that (a)-(c) are cited from [12]. horse animation (soft-body) simplification is demonstrated. In this extreme, highly nonrigid deformation example, the proposed method can still preserve both the geometric and motion details well in the simplification process. The problem incurred by single connectivity will lead to bad approximations in certain frames, as mentioned in [11] and [12], frames which did not occur using the proposed method. For the dynamic connectivity approaches such as [11], [12], they attempt to change the connectivity to better fit the deforming surface and produce high quality approximation on each frame. However, the deforming meshes with non-consistent connectivity will cause unpleasant popping effects. Even though the temporal coherence is managed, the popping effect cannot be avoided. Therefore, the simplified animation results generated by the proposed method are more smooth and pleasing than that generated by [11], [12]. It implies that the least geometrical error does not mean it is the best solution. If the overall geometrical error is higher for an obtained simplification, it can be a good solution when the motion information is taken into account. For visual comparison, please see our accompanying video. ${ }^{1}$ We also show the quality and visual comparisons between our approach and several related approaches [15], [26] in Figs. 9 and 10 . We extend and apply the mesh saliency approach presented in [26] to the animating mesh simplification by taking the sum of edge-collapsing cost in all frames as the simplification metric [Fig. 10(f)]. We also slightly modify the simplification metric presented in [15] [Fig. 10(e)] for the maximum edge-collapsing cost over all frames [Fig. 10(d)]. We can see that the proposed method has better quality than these works, in terms of shape preservation and PSNR, on parts of the animating mesh that deform relatively more as compared to nonfeature parts of the mesh. Please see our accompanying video for visual comparison.

${ }^{1}$ Available: [Online]. http://graphics.csie.ncku.edu.tw/Paper_Video/TCSVT/ TCSVT_anim_mesh_2007.mp4

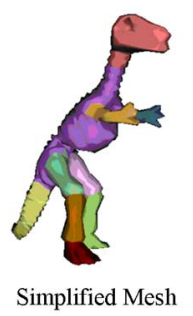

No. of face: 2000 $(10 \%)$

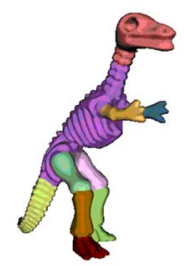
No. of face: 20000
Original Mesh

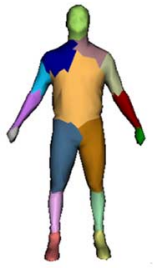

Simplified Mesh

No. of face: 1500 $(10 \%)$

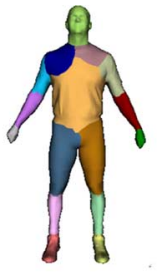

Original Mesh No. of face:

15000
Fig. 11. Partitioned results of Dinosaur and Runner deforming meshes.

\section{APPLications}

Many applications can benefit from the proposed methods. In this paper, we demonstrate two applications and their evaluated results: 1) deforming mesh decomposition and 2) animation compression and compact progressive representation for transmission.

\section{A. Efficient Deforming Mesh Decomposition}

Deforming mesh decomposition plays a critical role in computer graphics. Recently, many schemes [18], [24] concentrated on automatically partitioning the deforming mesh. However, these approaches are very time consuming due to the all-pairs shortest path finding and iterative face clustering requirements. Based on the proposed simplification scheme, the decomposition computation cost can be greatly reduced. We briefly introduce the workflow of our partitioning scheme as follows. First, we generate the coarser deforming mesh using the simplification approach mentioned in Section IV. Any deforming mesh decomposition approach can then be adopted here to decompose this simplified mesh. In this work, we use our previous deforming mesh partitioning method [18]. The next step is to map the decomposition boundary back to the original deforming mesh using projection. A common boundary smooth process is then applied to smooth out the decomposition boundaries. Fig. 11 shows the experimental results. The success of [18] is significantly related to deformation behaved by animating meshes. After simplification using the proposed method, the simplified animating meshes can still preserve motion features in the original meshes. Therefore, the proposed method can create good partitioning results based on simplified animating meshes. Table III shows the performance comparison between [18] and the proposed scheme. Obviously, the computation cost is reduced using the proposed scheme. 
TABLE III

Performance Comparison Between the Proposed Approach AND That of LeE et al. [18]

\begin{tabular}{|c|c|c|c|c|c|c|}
\hline & & Simplification & $\begin{array}{c}\text { Deformation } \\
\text { Analysis }\end{array}$ & Segmentation & Mapping & $\begin{array}{l}\text { Total } \\
\text { Time }\end{array}$ \\
\hline & [18] & -- & $163 \mathrm{sec}$. & $878 \mathrm{sec}$. & -- & $1041 \mathrm{sec}$. \\
\hline Dinosaur & $\begin{array}{c}\text { The proposed } \\
\text { method }\end{array}$ & $301 \mathrm{sec}$. & $12 \mathrm{sec}$. & $12 \mathrm{sec}$. & $1 \mathrm{sec}$. & $326 \mathrm{sec}$ \\
\hline & [18] & -- & $79 \mathrm{sec}$. & $629 \mathrm{sec}$. & - & $708 \mathrm{sec}$. \\
\hline Runner & $\begin{array}{c}\text { The proposed } \\
\text { method }\end{array}$ & $165 \mathrm{sec}$. & $12 \mathrm{sec}$. & $8 \mathrm{sec}$. & $1 \mathrm{sec}$. & $186 \mathrm{sec}$. \\
\hline
\end{tabular}

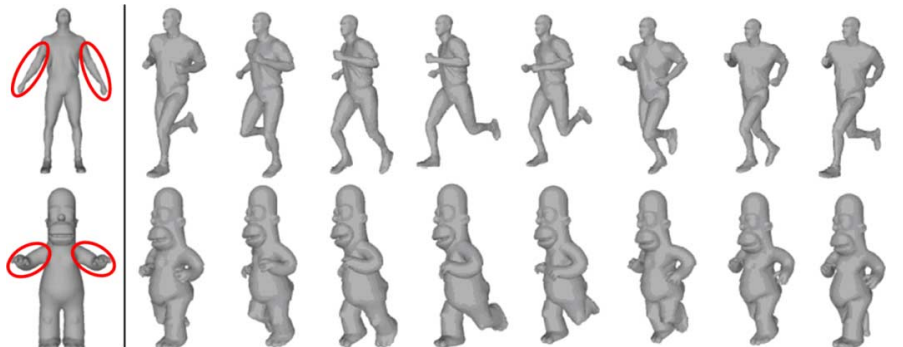

Fig. 12. Left: the reference pose. Top right: the source animation. Bottom right: the deformation transfer result generated using the proposed method.

As mentioned in [18], the deforming mesh decomposition result can be applied to the deformation transfer application [1]. Using the proposed decomposition scheme, we can also effectively handle the deformation transfer with difference reference poses. Fig. 12 shows the experimental result. The reference poses are different in the hands (the red ellipses on the left-hand side of Fig. 12).

\section{B. Animation Compression and Compact Progressive Representation}

In computer graphics, reducing the data complexity is a common way to overcome the rendering limitation or network bandwidth. Both the proposed key-frame extraction and simplification methods can reduce the data complexity of a given animation sequence. The extracted key-frames can be regarded as a compact representation in the temporal domain, as well as a simplified animation which can be regarded as a compact representation in the spatial domain. With the combination of these two approaches, the animation data size can be greatly reduced, as shown in Fig. 13. Furthermore, we also can construct a compact progressive animation representation for network transmission. Using the simplification approach, an animation can be represented as a progressive animation representation [4], that is, a base animation $\left(M_{0}^{0}, \cdots M_{n}^{0}\right)$ and a sequence of vertex split records $\left(v s p l_{0}^{0}, \cdots v s p l_{n}^{0}\right), \cdots,\left(v s p l_{0}^{k}, \cdots v s p l_{n}^{k}\right)$. With the extracted key-frames, we can replace the base animation using extracted key-frames and a set of weights for non-key-frames reconstruction. We can only store the vertex split records for key-frames. Therefore, the progressive animation representation is very compact as follows:

$$
\begin{array}{r}
\left\{\left(M_{\mathrm{key} 0}^{0}, \cdots, M_{\mathrm{key} m}^{0},\left(w_{i j}\right)\right),\left(v s p l_{\mathrm{key} 0}^{0}, \cdots v s p l_{\mathrm{key} m}^{0}\right), \cdots\right. \\
\left.\left(v s p l_{\mathrm{key} 0}^{k}, \cdots v s p l_{\mathrm{key} m}^{k}\right)\right\} \cdot
\end{array}
$$
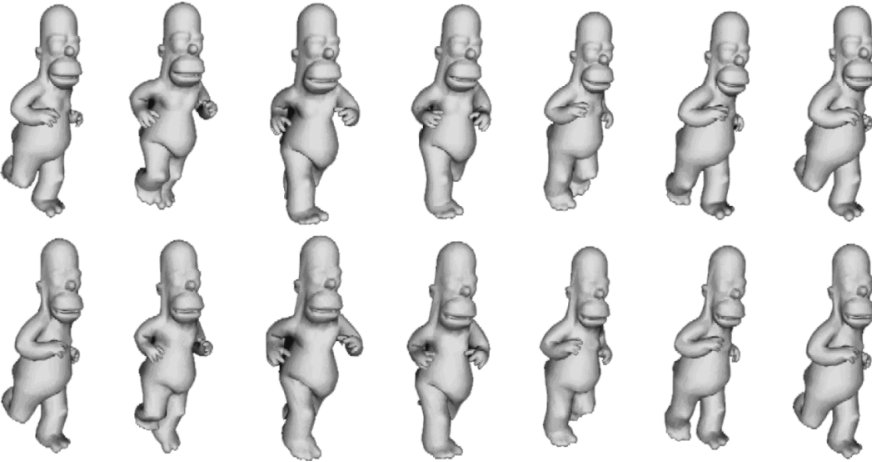

Fig. 13. Top row: the original animation (no. of face: 10202; no. of frames: 25 ). Bottom row: the animation generated by the proposed simplification and key-frame extraction approaches (no. of face: 3062; no. of key-frames: 6).

\section{CONCLUSION}

We have introduced novel methods for animation mesh simplification and key-frame extraction to produce a very compact representation for animating meshes. The proposed approaches are driven not only by the common geometric error analysis, but also by the deformation analysis of animating meshes. Therefore, the geometric features and the motion characteristics can be well-preserved in the simplified meshes, even for an extremely nonrigid animation. In addition, the simplified results will not have any annoying popping caused by changing connectivity/geometry, because single connectivity is used to represent all frames. In key-frame extraction, the deformation-driven GA method is proposed to solve the optimization search problem. The experimental results show that this new method is very efficient in searching for good key-frames while the quality is comparable to that of a state-of-the-art technique [8]. We also experimentally demonstrated the usefulness of the proposed methods to the relative applications: deforming mesh decomposition and compact progressive representation for animation transmission. The experimental results show that the proposed method can be successfully applied to these two applications.

\section{ACKNOWLEDGMENT}

The authors would like to thank the anonymous reviewers for their helpful comments that improved the paper.

\section{REFERENCES}

[1] R. W. Sumner and J. Popovic, "Deformation transfer for triangle meshes," ACM Trans. Graph. (SIGGRAPH 2004), vol. 23, no. 3, pp. 399-405, 2004. 
[2] R. W. Sumner, M. Zwicker, C. Gotsman, and J. Popovic, "Mesh-based inverse kinematics," in Proc. SIGGRAPH 2005, Aug. 2005, vol. 24, no. 3, pp. 488-495.

[3] M. Garland and P. S. Heckbert, "Surface simplification using quadric error metrics," in Proc. SIGGRAPH, 1997, pp. 209-216.

[4] H. Hoppe, "Progressive meshes," in Proc. SIGGRAPH, 1996, pp. $99-108$.

[5] N. Dimitrova, H.-J. Zhang, B. Shahraray, I. Sezan, T. Huang, and A. Zakhor, "Applications of video-content analysis and retrieval," IEEE Multimedia, vol. 9, no. 3, pp. 42-55, Jul.-Sept. 2002.

[6] Y. Gong and X. Liu, "Video summarization and retrieval using singular value decomposition," ACM Multimedia Syst. J., vol. 9, no. 2, pp. 157-168, Aug. 2003.

[7] S. Uchihashi, J. Foote, A. Girgensohn, and J. Boreczky, "Video manga: Generating semantically meaningful video summaries," in Proc. 7th ACM Int. Conf. Multimedia (Part 1), New York, 1999, pp. 383-392.

[8] K. S. Huang, C. F. Chang, Y. Y. Hsu, and S. N. Yang, "Key probe: A technique for animation keyframe extraction," Visual Comput., vol. 21, no. 8-10, pp. 532-541, 2005.

[9] I. S. Lim and D. Thalmann, "A key-posture extraction out of human motion data," in Proc. 23rd Ann. Int. Conf. IEEE Eng. Med. Biol. Soc., 2001, pp. 1167-1169.

[10] M. J. Park and S. Y. Shin, "Example-based motion cloning," Comput. Animat. Virtual Worlds, vol. 15, no. 3-4, pp. 245-257, 2004.

[11] S. Kircher and M. Garland, "Progressive multiresolution meshes for deforming surfaces," in Proc. ACM/Eurographics Symp. Comput. Animation, 2005, pp. 191-200.

[12] F.-C. Huang, B.-Y. Chen, and Y.-Y. Chuang, "Progressive deforming meshes based on deformation oriented decimation and dynamic connectivity updating," in Proc. ACM SIGGRAPH/Eurographics Symp. Comput. Animation, Vienna, Austria, 2006, pp. 53-62.

[13] A. Shamir and A. Pascucci, "Temporal and spatial level of details for dynamic meshes," in Proc. ACM Symp. Virtual Reality Software Technol., 2001, pp. 77-84.

[14] A. Shamir, V. Passucci, and C. Bajaj, "Multiresolution dynamic meshes with arbitrary deformations," in Proc. Visualization Conf., 2000, pp. 423-430.

[15] A. Mohr and M. Gleicher, Deformation Sensitive Decimation Univ. of Wisconsin, Madison, Rep. 4/7/2003.

[16] C. Decoro and S. Rusinkiewicz, "Pose-independent simplification of articulated meshes," in Proc. Symp. Interactive 3-D Graphics and Games, Washington, DC, Apr. 2005, pp. 17-24.

[17] D. L. James and C. D. Twigg, "Skinning mesh animations," in Proc. SIGGRAPH, Aug. 2005, vol. 24, no. 3, pp. 399-407.

[18] T. Y. Lee, Y. S. Wang, and T. G. Chen, "Segmenting a deforming mesh into near-rigid components," Vis. Comput., vol. 22, no. 9, pp. 729-739, Sep. 2006.

[19] T. Popa, D. Julius, and A. Sheffer, "Material aware mesh deformations," in Proc. IEEE Int. Conf. Shape Modeling Appl., Jun. 2006.

[20] F. Liu, Y. Zhuang, F. Wu, and Y. Pan, "3D motion retrieval with motion index tree," Comput. Vis. Image Understand, vol. 92, no. 2, pp. 265-284, 2003.

[21] A. Hyvarinen, J. Karhunen, and E. Oja, Independent Component Analysis. New York: Wiley, 2001.

[22] I. T. Jolliffe, Principal Component Analysis. Berlin, Germany: Springer-Verlag, 2002.

[23] M. Alexa and W. Muller, "Representing animations by principal components," Comput. Graphics Forum, vol. 19, no. 3, pp. 411-418, Aug. 2000.

[24] T. Y. Lee, P. H. Lin, S. U. Yan, and C. H. Lin, "Mesh decomposition using motion information from animation sequences," Comput. Animation Virtual Worlds, vol. 16, no. 3-4, pp. 519-529, Jul. 2005.

[25] L. Itti, C. Koch, and E. Niebur, "A model of Saliency based visual attention for rapid scene analysis," IEEE Trans. Pattern Anal. Mach. Intell., vol. 20, no. 11, pp. 1254-1259, Nov. 1998.
[26] C. H. Lee, A. Varshney, and D. W. Jacobs, "Mesh Saliency," ACM Trans. Graphics, vol. 24, no. 3, pp. 659-666, 2005.

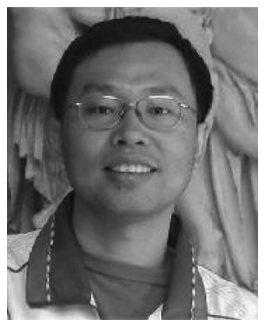

Tong-Yee (Tony) Lee received the Ph.D. degree in computer engineering from Washington State University, Pullman, in 1995.

He is currently a Professor with the Department of Computer Science and Information Engineering, National Cheng-Kung University, Tainan, Taiwan, R.O.C. His current research interests include computer graphics, nonphotorealistic rendering, image-based rendering, visualization, virtual reality, surgical simulation, medical visualization and medical systems, and distributed and collaborative virtual environment. He leads a Computer Graphics Group/Visual System Lab at National Cheng-Kung University.

Prof. Lee is a member of the ACM. He is serving as an Associate Editor for the IEEE TRANSACTIONS ON INFORMATION TECHNOLOGY IN BIOMEDICINE from 2000 to 2010. He is also on the Editorial Advisory Board of the journal Recent Patents on Engineering, editor for the Journal of Information Science and Engineering and region editor for the Journal of Software Engineering.

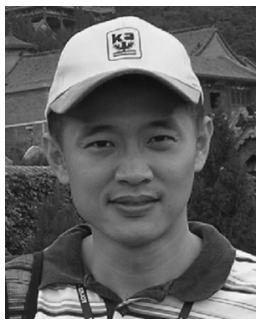

Chao-Hung Lin was born in Koushung, Taiwan, R.O.C., in 1973. He received the B.S. degree in computer science and engineering from $\mathrm{Fu}-\mathrm{Jen}$ University, Taipei, Taiwan, R.O.C., in 1997, and the M.S. and Ph.D. degrees in computer engineering from National Cheng-Kung University, Tainan, Taiwan, R.O.C., in 1998 and 2004, respectively.

$\mathrm{He}$ is currently an Assistant Professor with the Department of Geomatics, National Cheng-Kung University. His current research interests include computer graphics, image processing, visualization,

and modeling.

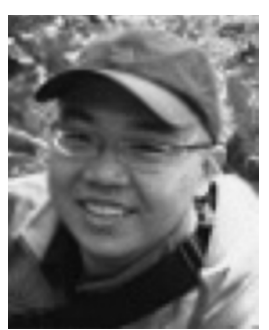

Yu-Shuen Wang received the B.S. degree from National Cheng Kung University, Tainan, Taiwan, R.O.C., in 2004. He is currently working toward the $\mathrm{Ph}$.D. degree in the Department of Computer Science and Information Engineering, National Cheng-Kung University, Tainan.

His research interests include computer graphics, mesh segmentation, skeletonization, and computer animation.

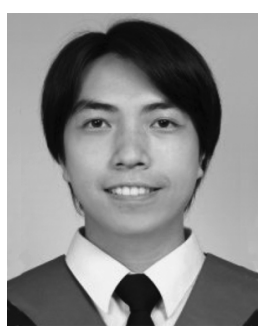

Tai-Guang Chen received the B.S. degree from National Sun Yat-sen University, Kaohsiung, Taiwan, R.O.C., in 2004. He is currently working toward the M.S. degree in the Department of Computer Science and Information Engineering, National Cheng-Kung University, Tainan, Taiwan, R.O.C.

His research interests include computer graphics, mesh segmentation, and computer animation. 